\title{
Control of a virtual ambulation influences body movement and motion sickness
}

\author{
Yi-Chou Chen ${ }^{*} \quad$ Xiao Dong ${ }^{*}$ Jens Hagstrom ${ }^{*}$ Thomas A. Stoffregen \\ (*) University of Minnesota, USA \\ E-mail:tas@umn.edu
}

\begin{abstract}
Drivers typically are less susceptible to motion sickness than passengers. The influence of vehicle control has theoretical implications for the etiology of motion sickness, and has practical implications for the design of virtual environments. In the present study, participants either controlled or did not control a nonvehicular virtual avatar (i.e., an ambulatory character in a console video game). We examined the incidence of motion sickness and patterns of movement of the head and torso as participants either played or watched the game.

Motion sickness incidence was lower when controlling the virutal avatar than when watching an avatar that was controlled by someone else. Patterns of head and torso movement differed between particpants who did and did not control the avatar. Indepenently, patterns of movement differed between participants who reported motion sickness and those who did not.

The results suggest that motion sickness is influenced by control of stimulus motion, whether that motion arises from a vehicle or from any other source. We consider implications for the design of humancomputer interfaces.
\end{abstract}

\section{Introduction}

In vehicular travel, motion sickness is more common among passengers, and less common among drivers [1]. This effect has long been acknowledged as having significance for our understanding of motion sickness etiology [2,3]. Recent research has revealed that this issue applies also to the control of virtual vehicles: Drivers of virtual vehicles are less likely than passengers to report motion sickness [4]. The explanation for the "control effect" may lie in relations between vehicle motion and control of the body.

Extensive research has shown that the subjective symptoms of motion sickness are preceded by changes in postural activity (movement of the body) that are unique to individuals who later report becoming motion sick. This effect has been observed in laboratory devices, virtual environments, flight simulators, and console video games [5-8]. Interestingly, in console video games postural instability and motion sickness are common among individuals who control the game (i.e., players), and occur in games that depict ambulation (i.e., walking or running) rather than vehicular travel [8]. These findings raise a new question: Is motion sickness etiology influenced by control of vehicles, per se? Or, is motion sickness etiology influenced by control of stimulus motion, regardless of whether any vehicle (physical or virtual) is involved?

In the present study, we investigated motion sickness incidence and postural activity when participants either controlled or did not control ambulatory activity in a console video game.

\section{Method}

\section{Participants}

Twenty-three college students participated as volunteers or in exchange for course credit. Each reported that they were in good health and had no history of vestibular disorders, dizziness, or falls.

\section{Apparatus}

We used a standard Xbox 360 game system, with a standard gamepad controller.

Movement data were collected using a magnetic tracking system (FasTrak, Polhemus Inc., Colchester VT, USA).

\section{Procedure}

Participants were run individually. We used a yoked-control design. Odd-numbered participants

This is an Open Access article distributed under the terms of the Creative Commons Attribution-Noncommercial License 3.0, which permits unrestricted use, distribution, and reproduction in any noncommercial medium, provided the original work is properly cited. 
played the game, while even-numbered participants watched a recording of the previous participant's game. We used Call of Duty, a first person shooter game in which the player, controlling an avatar, attempts to find and kill enemies. Each Player's performance was recorded and (later) replayed for the corresponding Viewer. Participants were exposed to the game for a maximum of 50 mintues. Each participant was instructed to discontinue immediately if they experienced any symptoms of motion sickness, however mild.

Participants sat on a stool $1.5 \mathrm{~m}$ in front of the display (Figure 1). The game was displayed on a 1.65 $\mathrm{m}$ (diagonal) plasma flat screen display unit, with builtin stereo speakers. One sensor from the magnetic tracking system was attached to a bicycle helmet worn be each participant, while another was attached to the torso at the level of the $7^{\text {th }}$ cervical vertebra. Movement data were sampled at $60 \mathrm{~Hz}$ continuously throughout exposure to the game.

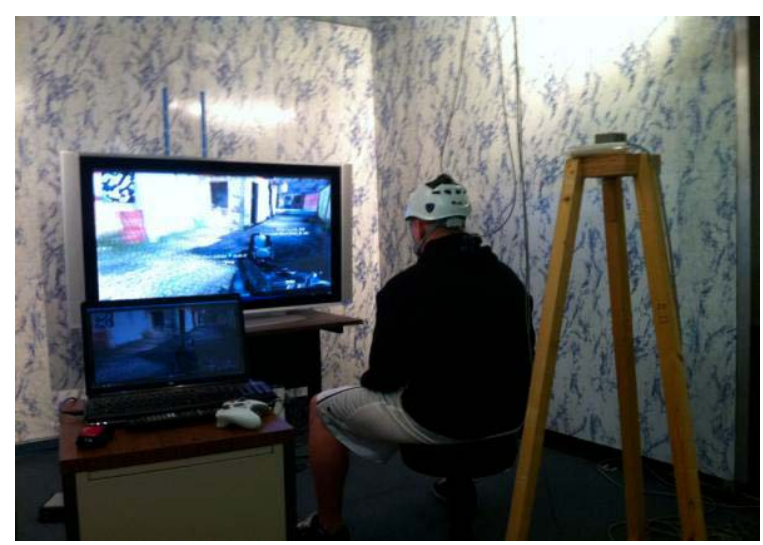

Figure 1. Experimental setting.

At the end of game exposure (completion of the 50 minute session or discontinuation, whichever came first), each participant answered a forced-choice, yes/no question about their motion sickness status. Participants were assigned to Sick and Well groups on the basis of these statements. For movement data, we evaluated the standard deviation of position, with separate analyses for movement in the AP (anteriorposterior and ML (mediolateral) axes.

\section{Results}

Eight of 23 participants reported motion sickness (35\%). Among Players, 2 of 11 (18\%) reported sickness, while among Viewers the incidence was 50\% (6/12); this difference was significant. Overall, Players moved more than Viewers (Figure 2). Movement differed between Sick and Well, as a function of time (Figure 3).

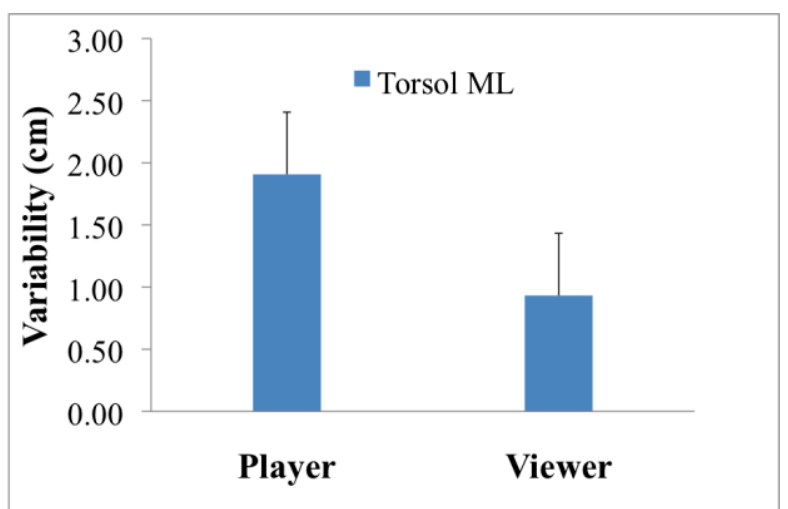

Figure 2. Positional variability of torso in the ML axis, for Players and Viewers.

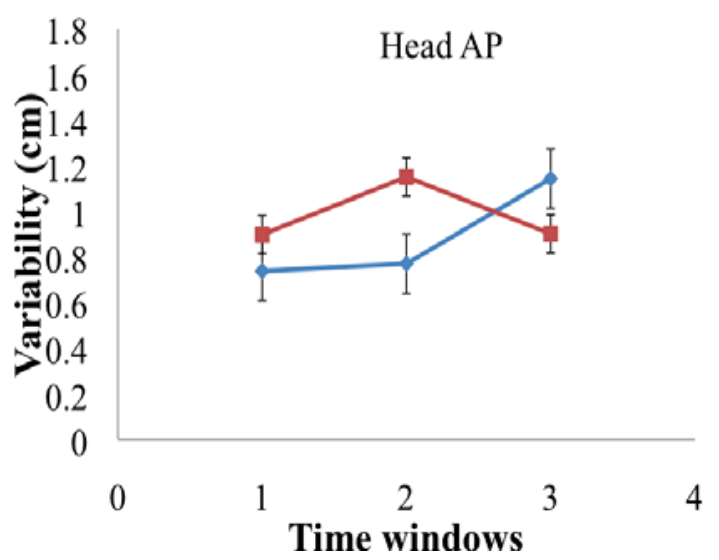

Figure 3. Positional variability of the head in the AP axis for Sick and Well groups for three 2-minute time windows during game exposure. Blue: Sick. Red: Well.

\section{Discussion}

Seated participants were exposed to a console video game featuring ambulation in a "first person shooter" context. Participants either played the game (Players) or viewed recorded game sessions (Viewers). There were three important results.

First, Viewers were more likely than Players to report motion sickness. This result reveals that motion sickness etiology is related to control of virtual movement in general, and is not limited to the control of virtual vehicles as such.

Second, as a group, Players moved more than Viewers. This result is not surprising and replicates a 
similar finding with drivers and passengers of virtual vehicles [4].

Finally, over time movement tended to be stable among Well participants, while among participants who reported motion sickness movement tended to increase over time during game exposure. This result resembles similar findings from studies of video games [8], virtual environments [6], and vehicle simulators [7].

Overall, the results demonstrate that the etiology of motion sickness is powerfully influenced by whether a person has control over the motion stimulus. The results suggest that viewers of virtual environments are at greater risk of motion sickness than individuals who control the displays. This effect has implications for interfaces that are intended to be viewed by multiple individuals, such as drivers and passengers, managers and workers, or teachers and students.

\section{References}

[1] A. Rolnick and R. E. Lubow, R. E. Why is the driver rarely motion sick? The role of controllability in motion sickness. Ergonomics, 34, 867-879, 1993.

[2] I. P. Howard and W. B. Templeton, W. B. Human spatial orientation. New York: Wiley, 1966.

[3] J. T. Reason and J. J. Brand. Motion sickness. London: Academic Press, 1975.
[4] X. Dong, K. Yoshida, and T. A. Stoffregen, T. A. Control of a virtual vehicle influences postural activity and motion sickness. Journal of Experimental Psychology: Applied, in press, 2011.

[5] C. T. Bonnet, E. M. Faugloire, M. A. Riley, B. G. Bardy, and T. A. Stoffregen. Motion sickness preceded by unstable displacements of the center of pressure. Human Movement Science, 25, 800-820, 2006.

[6] S. Villard, M. B. Flanagan, G. Albanese, and T. A. Stoffregen. Postural instability and motion sickness in a virtual moving room. Human Factors, 50, 332-345, 2008.

[7] T. A. Stoffregen, L. J. Hettinger, M. W. Haas, M. Roe, and L. J. Smart. Postural instability and motion sickness in a fixed-base flight simulator. Human Factors, 42, 458-469, 2000.

[8] T. A. Stoffregen, E. Faugloire, K. Yoshida, M. Flanagan, and O. Merhi. Motion sickness and postural sway in console video games. Human Factors, 50, 322331,2008 